\title{
Mapping of Adult Plant Leaf Rust Resistance in Aus27506 and Validation of Underlying Loci by In-Planta Fungal Biomass Accumulation
}

\author{
Pakeerathan Kandiah ${ }^{1,+, \ddagger(D)}$, Mumta Chhetri ${ }^{1, \ddagger}$, Matthew Hayden ${ }^{2,3}$, Michael Ayliffe ${ }^{4}$, \\ Harbans Bariana ${ }^{1, *(D)}$ and Urmil Bansal ${ }^{1, *(D)}$ \\ 1 School of Life and Environmental Sciences, Faculty of Science, The University of Sydney Plant Breeding \\ Institute, 107 Cobbitty Road, Cobbitty, NSW 2570, Australia; pakeerathank@univ.jfn.ac.lk (P.K.); \\ Mumta.Chhetri@csiro.au (M.C.) \\ 2 Agriculture Victoria, Centre for AgriBioscience, AgriBio, 5 Ring Road, Bundoora, VIC 3083, Australia; \\ matthew.hayden@agriculture.vic.gov.au \\ 3 School of Applied Systems Biology, La Trobe University, Bundoora, VIC 3086, Australia \\ 4 CSIRO Agriculture Flagship, GPO Box1600, Canberra, ACT 2601, Australia; Michael.Ayliffe@csiro.au \\ * Correspondence: harbans.bariana@sydney.edu.au (H.B.); urmil.bansal@sydney.edu.au (U.B.) \\ + Current address: Department of Agricultural Biology, The University of Jaffna, Aryviyal Nagar, \\ Kilinochchi 44000, Sri Lanka. \\ $\ddagger \quad$ These authors contributed equally to this work.
}

Received: 27 April 2020; Accepted: 20 June 2020; Published: 1 July 2020

\begin{abstract}
Among the rust diseases, leaf rust of wheat caused by Puccinia triticina, is the most prevalent worldwide and causes significant yield losses. This study aimed to determine the genomic location of loci that control adult plant resistance (APR) to leaf rust in the pre-Green Revolution landrace accession, Aus27506, from the "Watkins Collection". An Aus27506/Aus27229-derived F recombinant inbred line (RIL) population was screened under field conditions across three cropping seasons and genotyped with the iSelect 90K Infinium SNP bead chip array. One quantitative trait loci (QTL) on each of the chromosomes 1BL, 2B and 2DL explained most of the leaf rust response variation in the RIL population, and these were named QLr.sun-1BL, QLr.sun-2B and QLr.sun-2DL, respectively. QLr.sun-1BL and QLr.sun-2DL were contributed by Aus27506. QLr.sun-1BL is likely Lr46, while QLr.sun-2DL appeared to be a new APR locus. The alternate parent, Aus27229, carried the putatively new APR locus QLr.sun-2B. The comparison of average severities among RILs carrying these QTL in different combinations indicated that QLr.sun-2B does not interact with either of the other two QTL; however, the combination of QLr.sun-1BL and QLr.sun-2DL reduced disease severity significantly. In planta fungal quantification assays validated these results. The RILs carrying QLr.sun-1BL and QLr.sun-2DL did not differ significantly from the parent Aus27506 in terms of resistance. Aus27506 can be used as a source of adult plant leaf rust resistance in breeding programs.
\end{abstract}

Keywords: wheat; leaf rust; QTL mapping

\section{Introduction}

Leaf rust, caused by Puccinia triticina (Eriks and E. Henn), is an important disease of wheat. This rust pathogen is common across a range of geographical environments [1] and was introduced into Australia by early migrant settlers [2]. Several subsequent introductions, combined with the evolution of $P$. triticina $(\mathrm{Pt})$ pathotypes in Australia, have defeated many of the available leaf rust resistance genes. For example, after 25 years of deployment, $L r 24$ was overcome in South Australia in 2000 due to the evolution of virulence in Pt pathotype 104-1,2,3,(6),(7),11. One of the more recent exotic 
leaf rust incursions, Pt pathotype 104-1,3,4,6,7,8,9,10,12+Lr37 (which has combined virulence for the leaf rust resistance genes Lr1, Lr3a, Lr13, Lr14a, Lr15, Lr17a, Lr17b, Lr20, Lr26, Lr28, Lr27+Lr31 and Lr37) currently predominates in Australia [3]; (http://sydney.edu.au/agriculture/documents/pbi/cereal_rust_ report_2014.pdf).

The transfer of genetically diverse resistance genes in wheat cultivars is the most cost-effective way to control rust diseases [4]. Leaf rust resistance genes can be divided into two classes based on the plant growth stage at which resistance is expressed [5]. Most leaf rust resistance genes condition all stage resistance (ASR) that is effective against avirulent pathotypes throughout the plant growth [6]. ASR genes often confer high levels of resistance, but they can be rapidly defeated by pathogen evolution. In contrast, adult plant resistance (APR) genes, only provide resistance in post seedling stages. APR is typically partially effective and not associated with hypersensitive host cell death [4]. However, combinations of two or more APR genes can provide moderate levels of resistance and this type of resistance is assumed to be durable [7,8]. However, a few atypical APR genes also exist; for example, $L r 22 b$ is race-specific and expresses high levels of hypersensitive resistance at the adult plant stages suggesting that mechanistically it is more similar to ASR genes.

Over the last two decades, rust resistance breeding has reduced the deployment of ASR in favor of APRs and/or combinations of both types through marker-assisted selection [6]. APR expression under field conditions can be detected by different methods, such as the area under disease progress [9], size and number of uredinia produced during disease development and latent period $[10,11]$. These measurements are laborious and require specialized skills. To overcome the difficulties of these time-consuming disease assessment methods, Ayliffe et al. [12] introduced a simple and quick method to quantify the fungal biomass in plant tissues by the detection of chitin. Using this assay, the additive effects of different gene combinations of ASR and APR $(S r 33+S r 2, S r 2+S r 45)$ were compared with individual gene effects $(S r 33, S r 45$ and $S r 2)$. Similarly, the comparative effectiveness and additivity of the APR loci $L r 34 / Y r 18, L r 46 / Y r 29$ and $L r 67 / Y r 46$ was also examined [12].

Eighty quantitative trait loci (QTL) for leaf rust resistance have been mapped [13], and many have been detected in multiple studies. Both wheat landraces and close relative species of wheat have been used to discover new sources of disease resistance $[14,15]$. In this study, a pre-Green Revolution wheat landrace collected from France (Aus27506), that showed a susceptible response to leaf rust at the seedling stage, but exhibited adult plant resistance, was used for mapping putatively new source(s) of resistance.

\section{Materials and Methods}

\subsection{Development of Mapping Population}

The leaf rust resistant landrace Aus27506 was selected from the "Watkins Collection" [14] and crossed with the moderately susceptible landrace Aus27229. A recombinant inbred line (RIL) population consisting of 106 RILs $\left(\mathrm{F}_{6: 7}\right)$ was developed.

\subsection{Greenhouse Tests}

Aus27506 and Aus27229 were tested at the two-leaf stage with the Pt pathotypes 104-1,3,4,6,7,8,9,10,12+Lr37, 104-2,3,6,(7),12, 76-3,5,7,9,10,12,13+Lr37 and 10-1,3,9,10,11,12 and at the $4^{\text {th }}$ leaf stage with the $\mathrm{Pt}$ pathotype 104-1,3,4,6,7,8,9,10,12+Lr37 (https: //www.sydney.edu.au/content/dam/corporate/documents/sydney-institute-of-agriculture/research/ plant-breeding-and-production/cereal_rust_report_2016_14_3.pdf). The leaf rust inoculation, incubation and disease assessments were conducted according to McIntosh et al. [16].

\subsection{Field Evaluation}

Eight to ten seeds of each RIL and both parents were sown as hill-plots at the experimental sites Lansdowne (LDN), Karalee (KAR) and Horse research unit (HRU) of the University of Sydney Plant 
Breeding Institute (PBI), Cobbitty, during the 2014, 2015 and 2016 cropping seasons in two replications. The leaf rust spreader rows (a mixture of the susceptible genotypes MacKellar, Sonora, QAL2000, Morocco, Yitpi, Westonia and Stylet) were sown after every fifth row. In addition, each block of $35 \times 2$ experimental hill-plots was surrounded by a $30 \mathrm{~cm}$ susceptible spreader row. Leaf rust epidemic was created by spraying mixtures of urediniospores (Pt isolates 104-1,3,4,6,7,8,9,10,12+Lr37, 104-2,3,6,(7),12, $76-3,5,7,9,10,12,13+\operatorname{Lr} 37$ and 10-1,3,9,10,11,12) suspended in light mineral oil on the spreaders using an ultra-low-volume applicator (Microfit ${ }^{\mathrm{TM}}$, Micron Sprayer Ltd. Herefordshire, U.K.). The experimental area was irrigated using a sprinkler irrigation system when required to enhance crop growth and to create congenial conditions for rust development.

Adult plant leaf rust responses were scored from flag leaf initiation to grain filling at weekly intervals based on a 1-9 scale [5]. The 1-9 scale was converted to a disease severity score [17] to compare responses of RILs carrying different combinations of the QTL for leaf rust resistance.

\subsection{Molecular Mapping}

\subsubsection{DNA Isolation and Quantification}

Genomic DNA was extracted from the RILs and parents using a modified CTAB method [18] and quantified using a Nanodrop ND-1000 spectrophotometer (Nanodrop Technologies, Wilmington, NC, USA).

\subsection{2. iSelect $90 \mathrm{~K}$ Infinium Bead Chip Array Genotyping}

The RIL population was genotyped at AgriBio, La Trobe University, Melbourne, using the iSelect 90K Infinium SNP bead chip array described by Wang et al. [19]. The RILs that did not produce high quality genotypic data ( $\geq 10 \%$ missing data) were excluded from downstream analyses. Monomorphic markers were also excluded. The remaining marker data were evaluated using Chi-squared analysis, and markers that deviated from a 1:1 segregation ratio were also discarded. Markers with $5 \%$ or fewer heterozygous calls were retained to avoid the false purging of heterozygous loci [20].

\subsubsection{Linkage Map Construction and QTL Analysis}

MapManager version QTXb20 [21] was used for the genetic linkage map construction. The Kosambi mapping function [22] was used to convert recombination fractions into centiMorgans (cM). The redundant markers were excluded using the command "hide redundant loci", option and the phenotypic data were imported into MapManager. QTL cartographer [23] was used for composite interval mapping (CIM) based on 1000 permutations.

\subsubsection{Genotyping with Markers Linked with Known APR Genes}

To screen the parental lines for the presence of the previously characterised leaf rust APR genes, the following markers were tested; csLV34 for the Lr34 gene [24], SNPLr46G22 for the Lr46 gene (Lagudah unpublished) and csGS, cs7BLNLRR and Psy1-1 for the Lr68 gene [25]. These markers were amplified using standard PCR conditions, except for the KASP marker SNPLr46G22, for which the KASP assay described in Chhetri et al. was used [17]. The markers revealing polymorphism between the parents were tested on the entire RIL population and incorporated into the genetic map.

\subsection{Statistical Analysis}

Chi-squared analysis was used to test the goodness of fit of the observed segregation to the expected genetic ratios. Wright's formula [26] was used to estimate the number of adult plant leaf rust resistance loci segregating in the Aus27506/Aus27229 RIL population. Pearson's correlation coefficients were calculated among the different leaf rust response data sets using the function PROC CORR in SAS software version 9.4 (SAS Institute Inc., Cary, NC, USA). The least significant difference (LSD) was calculated to compare the mean disease severity of the RILs carrying different combinations of QTL. 


\subsection{Fungal Quantification Using Chitin Assay}

\subsubsection{Sample Collection}

Three leaf rust infected flag leaves, each from Aus27506, Aus27229 and RILs carrying different QTL combinations, were harvested from the field. An un-inoculated Aus27506 from the greenhouse was used as a negative control. The samples were weighed, cut into three-centimeter pieces and placed into $50 \mathrm{~mL}$ falcon screw cap tubes filled with $1 \mathrm{M} \mathrm{KOH}$ containing $0.1 \%$ Silwet. The samples from each genotype were replicated four times.

\subsubsection{Wheat Germ Agglutinin Chitin Assay (WAC)}

The fungal chitin was quantified in these samples using the method described by Ayliffe et al. [12]. Briefly, each sample was autoclaved at $121^{\circ} \mathrm{C}$ and 15 psi for $20 \mathrm{~min}$ with loosened caps. The $\mathrm{KOH}$ solution was decanted, and the samples were washed twice with $50 \mathrm{mM}$ Tris- $\mathrm{HCl}$ pH 7.0 (Tris buffer) and left to neutralize in this buffer for at least $20 \mathrm{~min}$. The tissue was then resuspended in Tris buffer at $200 \mathrm{mg} / \mathrm{mL}$ fresh weight and homogenized with a probe sonicator. The homogenates $(200 \mu \mathrm{L})$ were aliquoted into $0.5 \mathrm{~mL}$ PCR tubes with four replicates per sample, and $10 \mu \mathrm{L}$ of a $1 \mathrm{mg} / \mathrm{mL}$ solution of wheat germ agglutinin-fluorescein isothiocyanate (WGA-FITC) was added to each tube. After $15 \mathrm{~min}$, the staining tubes were centrifuged at $600 \mathrm{rpm}$ and the supernatant was removed from each sample. The samples were washed three times with $200 \mu \mathrm{L}$ of Tris buffer and finally resuspended in $100 \mu \mathrm{L}$ of Tris buffer. The fluorescence was quantified in a fluorometer using $485 \mathrm{~nm}$ absorption and $535 \mathrm{~nm}$ emission wavelengths for $1.0 \mathrm{~s}$.

\section{Results}

\subsection{Greenhouse Tests}

Aus27506 and Aus27229 were susceptible [infection type (IT) 3+] at the two and three leaf stages against $\mathrm{Pt}$ pathotypes 104-1,3,4,6,7,8,9,10,12+Lr37, 104-2,3,6,(7),12, 76-3,5,7,9,10,12,13+Lr37 and 10-1,3,9,10,11,12 (https://www.sydney.edu.au/content/dam/corporate/documents/sydney-institute-ofagriculture/research/plant-breeding-and-production/cereal_rust_report_2016_14_3.pdf). The testing at the fourth leaf stage of these parental lines also showed susceptible IT 3+ against the Pt pathotype $104-1,3,4,6,7,8,9,10,12+\operatorname{Lr} 37$. These results indicated the absence of seedling and intermediate stage leaf rust resistance in both parents.

\subsection{Genotyping with Markers Linked with Known Genes}

The genotyping of the parental lines with the marker csLV34 indicated the absence of Lr34. The marker SNPLr46G22 produced the same allele in both parents, suggesting the presence of $L r 46$. The markers csGS, cs7BLNLRR and Psy1-1 were monomorphic between parents and produced an Lr68-specific amplicon, indicating the presence of $L r 68$ in both parents.

\subsection{Field Tests}

On a 1-9 scale, adult plant leaf rust response of Aus27506 varied from 3 (resistant to moderately resistant) to 4 (moderately resistant leaf rust response), whereas Aus27229 showed moderate susceptibility (score 6) across all the data sets over three years. The adult plant leaf rust responses among the RILs varied from 2 to 6 (Figure 1). The analysis of the leaf rust response variation among the Aus27506/Aus27229 RIL population using Wright's formula estimated the involvement of two to three resistance loci (2.42, 2.38 and 2.42 in the 2014-LDN, 2015-KAR and 2016-HRU experiments, respectively). The leaf rust responses across seasons were significantly correlated, and the Pearson correlation coefficients between data sets varied from 0.5 to 0.6 at $P<0.001$. 


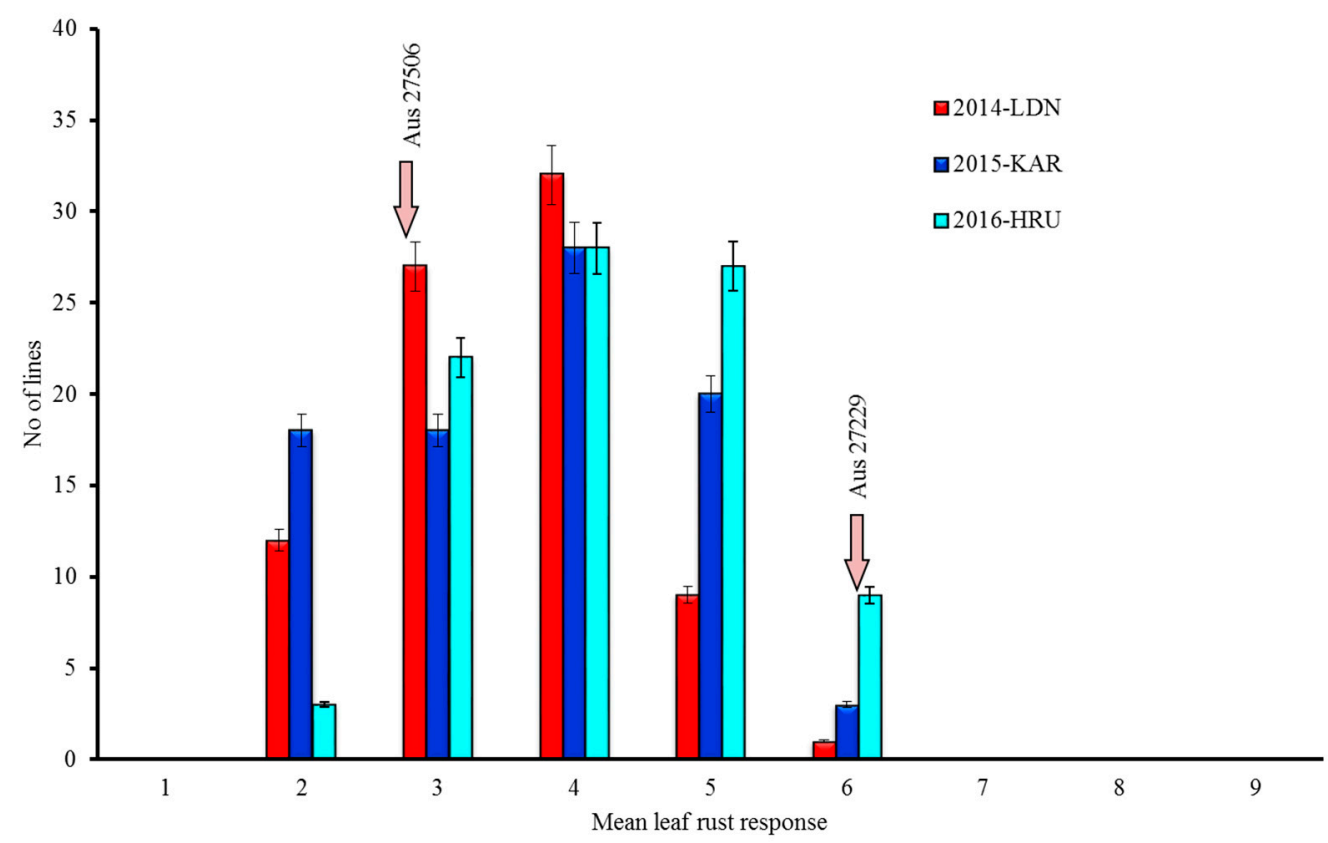

Figure 1. Leaf rust response variation among the Aus27506/Aus27229 recombinant inbred lines (RILs) when tested under field conditions. LDN: Lansdowne; KAR: Karalee; HRU: Horse research unit.

\subsection{Linkage Map Construction}

The Aus27506/Aus27229 RIL population was genotyped using a 90K wheat Infinium SNP bead chip array. The fifteen RILs with poor genotype calling were discarded, and a linkage map consisting of 2334 SNP showing 1:1 segregation was generated from 91 RILs. These markers formed 36 discrete linkage groups representing the 21 wheat chromosomes. The Aus27506/Aus27229 linkage map covered $6327.5 \mathrm{cM}$ with an average marker density of $2.71 \mathrm{cM}$. The total map length of the "A" genome was $2485.6 \mathrm{cM}$ with 951 markers, the " $\mathrm{B}$ " genome was $2915.7 \mathrm{cM}$ with 1130 markers, and the " $\mathrm{D}$ " genome was $926.2 \mathrm{cM}$ with 253 markers. The average marker densities of the " $A$ ", " $B$ " and " $D$ " genomes were $2.61 \mathrm{cM}, 2.58 \mathrm{cM}$ and $3.66 \mathrm{cM}$, respectively. The number of polymorphic markers for the " $\mathrm{B}$ " genome was the highest and for the D genome, the lowest.

\subsection{QTL Analysis}

Composite interval mapping (CIM) performed using the QTL Cartographer for the Aus27506/Aus27229 RIL population identified three QTL for leaf rust resistance on chromosomes 1BL, 2B and 2DL. These QTL were named QLr.sun-1BL, QLr.sun-2B and QLr.sun-2DL.

QLr.sun.1BL, contributed by Aus27506, explained $22 \%, 18 \%$ and $11 \%$ of the phenotypic variation, with logarithm of the odds (LOD) scores of 6.9, 4.76 and 2.7 in the 2014-LDN, 2015-KAR and 2016-HRU data sets, respectively. The QTL peaked at marker IWA8332, located on the long arm of the chromosome 1B, and it was flanked by the markers IWB74914 (IWGSC_RefSeq_v1.0 667,717,100 bp) and IWB72835 $(679,898,801$ bp) (Table 1; Figure 2a).

The leaf rust QTL on chromosome 2B (QLr.sun-2B) explained 6-12\% of the phenotypic variation and was derived from Aus27229. This QTL was statistically significant in the 2014-LDN and 2015-KAR data sets, with LOD scores of 3.53 and 2.98, respectively. QLr.sun-2B peaked at IWB63020, with the QTL interval defined by markers IWB68511 (313,499,584 bp) and IWB16756 (532,502,609 bp) (Table 1; Figure 2b).

Aus27506 contributed QLr.sun-2DL and it peaked at IWB64805. It explained 6-19\% of the phenotypic variation, with LOD values ranging from 2.3 to 4.89 across years/sites (Table 1; Figure 2c). QLr.sun-2DL spanned from IWB25696 (518,808,347 bp) to IWB23831 (518,808,247 bp). 
Table 1. Leaf rust quantitative trait loci (QTL) detected in the Aus27506/Aus27229 RIL population.

\begin{tabular}{ccccccc}
\hline QTL & Season/Site & Peak Marker & Flanking Markers & LOD & $\mathbf{R}^{\mathbf{2}}$ & Parent \\
\hline QLr.sun-1BL & 2014-LDN & IWA8332 & IWB74914-IWB72835 & 6.90 & 22 & Aus27506 \\
& 2015-KAR & IWA8332 & IWB74914-IWB72835 & 4.76 & 18 & Aus27506 \\
& 2016-HRU & IWA8332 & IWB74914-IWB72835 & $2.70^{\text {ns }}$ & 11 & Aus27506 \\
QLr.sun-2B & 2014-LDN & IWB63020 & IWB68511-IWB16756 & 3.53 & 12 & Aus27229 \\
& 2015-KAR & IWB63020 & IWB68511-IWB16756 & 2.98 & 09 & Aus27229 \\
& 2016-HRU & IWB63020 & IWB68511-IWB16756 & $1.48^{\text {ns }}$ & 06 & Aus27229 \\
QLr.sun-2DL & 2014-LDN & IWB64805 & IWB25696-IWB23831 & 4.89 & 19 & Aus27506 \\
& 2015-KAR & IWB64805 & IWB25696-IWB23831 & $2.30^{\text {ns }}$ & 06 & Aus27506 \\
& 2016-HRU & IWB64805 & IWB25696-IWB23831 & 3.40 & 12 & Aus27506 \\
\hline
\end{tabular}

RIL: recombinant inbred line; LDN: Lansdowne; KAR: Karalee; HRU: Horse research unit; ns: non-significance.

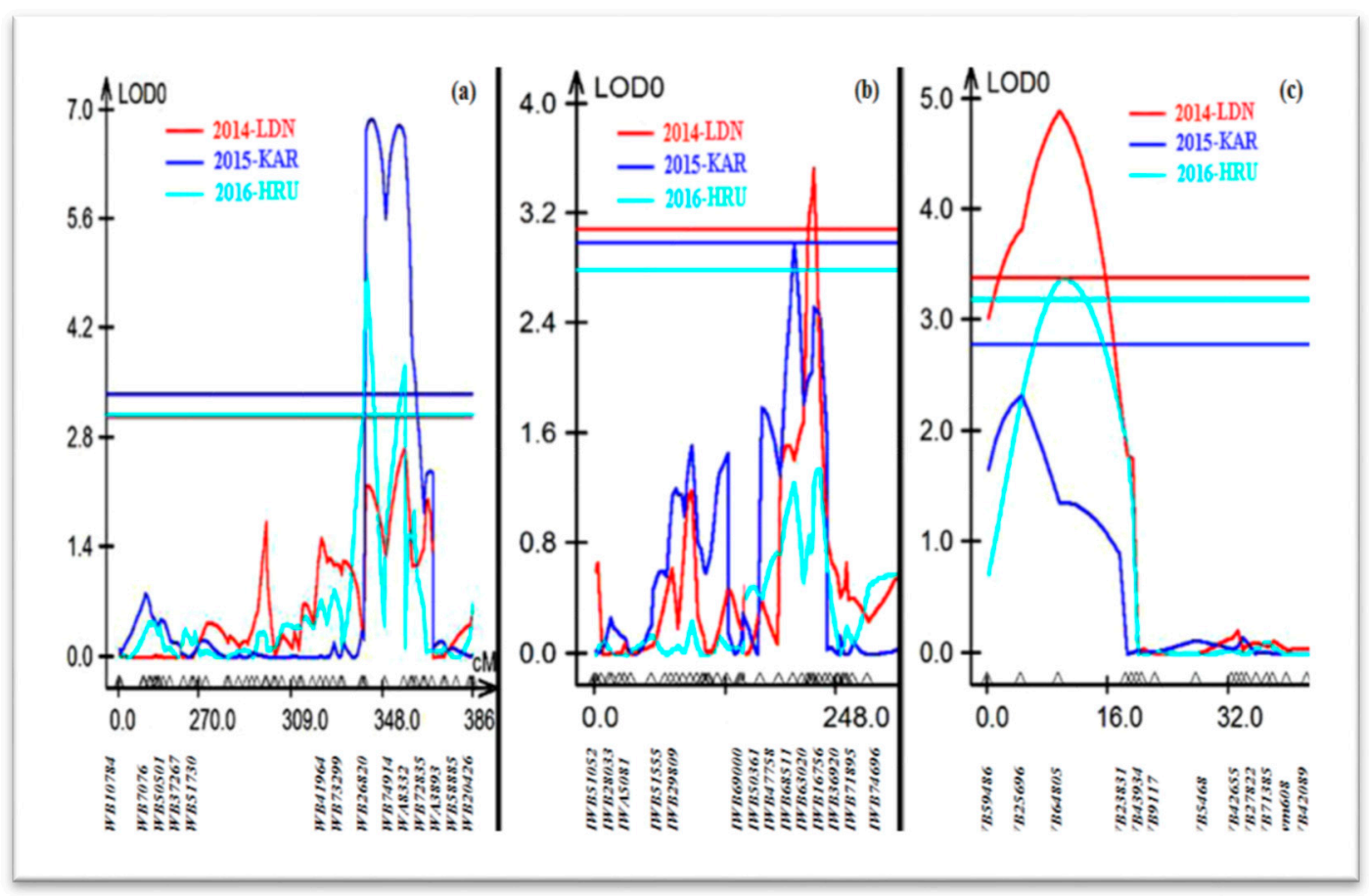

Figure 2. Leaf rust resistance QTL detected on chromosomes (a) 1BL, (b) 2B and (c) 2DL among Aus27506/Aus27229 RIL population.

\section{Interaction among QTL}

\subsection{Average Disease Severity among RILs with Different Combinations of QTL}

To study the interaction among the different QTL identified in this study, the RILs were categorized based on their QTL peak marker alleles and average disease severity scores. When QLr.sun-2B, QLr.sun-1BL and QLr.sun-2DL were present individually, the average leaf rust severities over the three field seasons were $32.0 \%, 19.1 \%$ and $18.3 \%$, respectively (Table 2). The phenotypic effects of QLr.sun-2DL and QLr.sun-1BL were statistically similar, whereas QLr.sun-2B showed significantly more disease severity. 
Table 2. Mean leaf rust severities of Aus27506/Aus27229 RILs carrying different QTL combinations.

\begin{tabular}{lcccc}
\hline \multicolumn{5}{c}{ Mean Leaf Severities } \\
\hline \multicolumn{1}{c}{ QTL } & 2014-LDN & 2015-KAR & 2016-HRU & Average \\
\hline QLr.sun-1BL & $18.2^{\mathrm{b}}$ & $20.00^{\mathrm{b}}$ & $19.20^{\mathrm{b}}$ & $19.13^{\mathrm{b}}$ \\
QLr.sun-2B & $31.00^{\mathrm{c}}$ & $28.00^{\mathrm{c}}$ & $37.00^{\mathrm{c}}$ & $32.00^{\mathrm{c}}$ \\
QLr.sun-2DL & $17.00^{\mathrm{b}}$ & $19.00^{\mathrm{b}}$ & $19.00^{\mathrm{b}}$ & $18.33^{\mathrm{b}}$ \\
QLr.sun-1BL+QLr.sun-2B & $17.50^{\mathrm{b}}$ & $18.46^{\mathrm{b}}$ & $19.00^{\mathrm{b}}$ & $18.32^{\mathrm{b}}$ \\
QLr.sun-2B+QLr.sun-2DL & $17.00^{\mathrm{b}}$ & $19.44^{\mathrm{b}}$ & $19.28^{\mathrm{b}}$ & $18.57^{\mathrm{b}}$ \\
QLr.sun-1BL+QLr.sun-2DL & $12.25^{\mathrm{a}}$ & $14.28^{\mathrm{a}}$ & $14.44^{\mathrm{a}}$ & $13.74^{\mathrm{a}}$ \\
QLr.sun-1BL+QLr.sun-2B+QLr.sun-2DL & $11.42^{\mathrm{a}}$ & $12.85^{\mathrm{a}}$ & $13.21^{\mathrm{a}}$ & $12.49^{\mathrm{a}}$ \\
RILs not carrying any QTL (Nil) & $42.00^{\mathrm{d}}$ & $41.00^{\mathrm{d}}$ & $40.83^{\mathrm{d}}$ & $40.87^{\mathrm{d}}$ \\
LSD & $4.05^{\mathrm{d}}$ & $3.00^{\mathrm{b}}$ & $2.98^{-}$ & 3.34 \\
\hline
\end{tabular}

Means of disease severity followed by different letters ( $a, b, c$ and $d)$ are significantly different based on LSD test at $P=0.05$. Same letter shows non-significance (ns). LSD: least significant difference.

The combination of $Q L$ r.sun-2B with either $Q L$ r.sun- $1 B L$ or $Q L$ r.sun-2DL did not significantly reduce leaf rust severity compared to the RILs carrying either of the later QTL singly. In contrast, rust severity in the RILs possessing both QLr.sun-1BL and QLr.sun-2DL was significantly lower than those RILs that carried them singly (Table 2). The three-QTL combination, QLr.sun-1BL+QLr.sun-2B+QLr.sun-2DL, produced an average disease severity of $12.49 \%$, which was similar to that with the two-QTL combination (QLr.sun-1BL+QLr.sun-2DL).

\subsection{Quantification of Fungal Biomass by Chitin Assay}

To assess the fungal biomass, infected flag leaves of the parents and representative RILs carrying different QTL combinations were collected and used for the chitin assay. Aus27506 and Aus27229 differed significantly for fungal biomass (Figure 3). The fungal growth of parent Aus27506, which carried QLr.sun-2DL and QLr.sun-1BL, was 61\% lower than that of Aus27229, which carried QLr.sun-2B. The RILs carrying all three QTL did not differ significantly for fungal biomass accumulation compared with Aus27506. RILs with QLr.sun-2DL + QLr.sun-1BL had more fungal colonization compared with Aus27506 and the RILs with all three QTL significantly reduced fungal growth compared to the RILs with the other two dual combinations (QLr.sun-1BL+QLr.sun-2B and QLr.sun-2B+QLr.sun-2DL) (Figure 3). The dual combinations involving QLr.sun-2B, contributed by Aus27229 and either QLr.sun-1BL or QLr.sun-2DL, showed similar levels of fungal growth, which were slightly less than that of Aus27229 (QLr.sun-2B). 


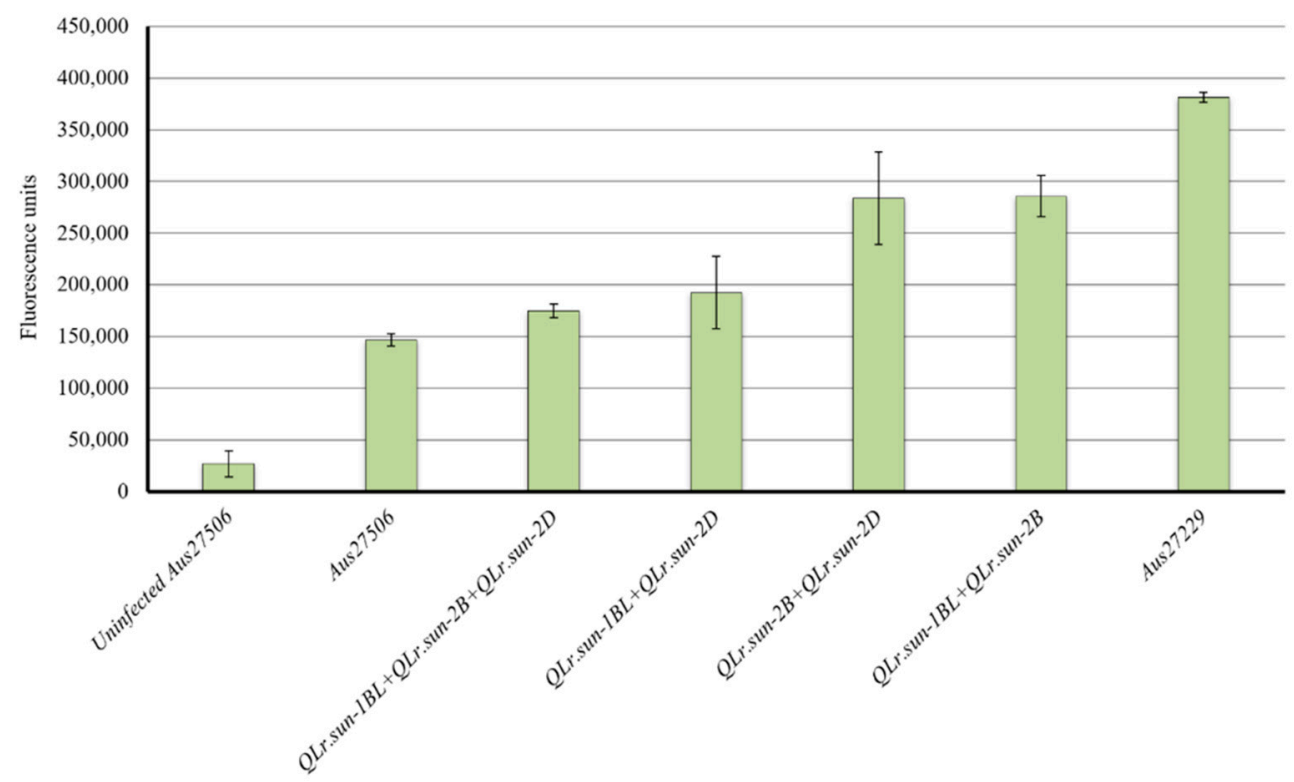

Figure 3. Quantification of rust growth in Aus27506, Aus27229 and RILs carrying different QTL combinations.

\section{Discussion}

The composite interval mapping of adult plant leaf rust response variation among an Aus27506/Aus27229 RIL population identified three QTL on chromosomes 1BL, 2B and 2DL, respectively. Chromosome 1B carries the formally designated leaf rust resistance genes $L r 26 / \mathrm{Yr} / \mathrm{Sr} 31$ [27], Lr33 [28], Lr44 [29], Lr46 [30], Lr51 [31], Lr55 [32], Lr71 [33] and Lr75 [34]. Of these genes, only Lr33 and Lr46 are located on the long arm. $L r 33$ is located $3 \mathrm{cM}$ distal to the centromere [28], and $L r 46$ is located in the most distal deletion bin (1BL-0.84-0.89) of the long arm [35]. QLr.sun-1BL, detected in this study, was located in the distal region of chromosome $1 \mathrm{BL}(137 \mathrm{cM}$ of a total map length of $174 \mathrm{cM})$. Screening with the Lr46-linked marker SNPLr46G22 did not differentiate the parents, presumably due to the non-diagnostic nature of this marker and, consequently, false positive amplification in Aus27229. QLr.sun-1BL explained $11-22 \%$ of the phenotypic variation, which is similar to that reported for Lr46 in other studies [13]. Furthermore, Lr46 appears to be better expressed in cooler climates [36]. The field trial sites in this study had an average temperature of 26.2 to $28.4{ }^{\circ} \mathrm{C}$ during the months of October and November in 2014,2015 and 2016 when the phenotypic scoring was performed, with occasional extremes of $35-38^{\circ} \mathrm{C}$ (http://www.bom.gov.au/climate/current/annual/nsw/summary.shtml\#recordsTmaxAvgHigh).

Taking into consideration the genetic map position of QLr.sun-1BL and percent phenotypic variation that it explained, we concluded that this QTL likely represents Lr46. Many other studies have also related QTL in chromosome 1BL to $\operatorname{Lr} 46[37,38]$.

The position of the QTL interval for QLr.sun-2B between 313,499,584 and 532,502,609 bp in the IWGSCv1.0 reference genome sequence indicated that it is located in the centromeric region. Lr48, an APR gene producing a low IT ( $23 \mathrm{~N}$ to $\mathrm{X})$ at the fourth leaf stage, co-segregated with the markers positioned at $59 \mathrm{cM}$ [39] in the published 90K SNP consensus map of Wang et al. [19]. However, the susceptibility of the parents in multi-pathotype greenhouse tests at different growth stages indicate that QLr.sun-2B does not correspond to Lr48.

Several QTL are reported on chromosome 2B, including QLr.ifa-2BS [40], QLr.ksu-2BS [41], QLr.sfrs-2BL [42], QLrlp.ous-2B [38], QLr.cimmyt-2BS [37], QLrlp.osu-2B [43] and QLr.osu-2B [44]. QLr.ifa-2BS is an allele of Lr13 from cultivar Forno and QLrlp.osu-2B, mapped closer to the centromere between the amplified fragment length polymorphism (AFLP) markers XCAGCGAT70 and XCATGATGC60. To demonstrate whether QLr.sun-2B represents a new APR locus, it will be 
important to enrich the target region with markers previously known to map onto chromosome $2 \mathrm{~B}$ and are associated with known leaf rust resistance loci.

The markers delineating the QLr.sun-2DL interval were positioned between 518,808,347 and $518,808,247$ bp in the IWGSC_RefSeq_v1.0 genome assembly. Taking into consideration the position of the linked markers, this QTL was located in the long arm of chromosome 2D. QLr.sfr-2DL [45], present on the long arm of chromosome $2 \mathrm{D}$, is reported to be closer to the centromere. It peaks at the simple sequence repeat (SSR) marker gwm539 and explains $11.4-12 \%$ of the phenotypic variation. The marker gwm539 was mapped at the $116 \mathrm{cM}$ position of the total $136 \mathrm{cM}$ length of chromosome 2D [46]. Hence, QLr.sun-2DL appears to be a new locus.

Of the three QTL detected in this study, QLr.sun-2DL and QLr.sun-1BL contributed more towards disease reduction compared to QLr.sun-2B. The interaction studies indicated that QLr.sun-2B does not interact with QLr.sun-2DL or QLr.sun-1BL to reduce disease severity. Many studies have reported disease severity to be lowered by combinations of APR genes; for example, Lr34 and Lr46, Lr34 and $L r 68$, and $L r 75$ and QLr.sfr-7BL showed enhanced resistance through additive gene action in different trials [34]. In-plant fungal quantification using the chitin assay is another measure to show the additive effect of resistance loci [12]. The RILs carrying two QTL combinations involving QLr.sun-2B showed more fungal biomass compared to the combination of QLr.sun-1BL and QLr.sun-2D. These results confirm conclusions drawn from the field disease severity score comparison of the RILs possessing different combinations of QTL.

The failure to observe segregation for a leaf rust response score beyond 6 suggests that Aus27506 and Aus27229 may have an APR locus in common. The monomorphism of the Lr68-linked marker is indicative of the presence of this gene in both parents. The development of single locus populations for QLr.sun-2B and QLr.sun-2DL is underway to enable the detailed mapping of the regions carrying these loci and to identify closely linked markers for their marker-assisted pyramiding in wheat breeding programs.

Author Contributions: U.B. and H.B. identified material and made initial cross. M.C. developed R.I.L. population. P.K. and H.B. conducted greenhouse and field screening. M.H. and M.A. conducted S.N.P. genotyping in-planta fungal biomass accumulation assays, respectively. P.K. drafted the manuscript. U.B., H.B., M.H. and M.A. edited drafts. All authors have read and agreed to the published version of the manuscript.

Funding: US00063-Australian Cereal Rust Control Program-Durable genes.

Acknowledgments: The first author thanks the Australian Government for the award of International Postgraduate Research Scholarship and Australian Postgraduate Award to pursue Ph.D. studies at the University of Sydney and acknowledges the University of Jaffna for granting study leave. Financial support from the GRDC Australia is gratefully acknowledged.

Conflicts of Interest: The authors declare no conflict of interest.

\section{References}

1. Kolmer, J.; Chen, X.; Jin, Y. Diseases which challenge global wheat production-The wheat rusts. In Wheat Science and Trade; Carver, B.F., Ed.; Wiley-Blackwell: Hoboken, NJ, USA, 2009; pp. 89-124.

2. Watson, I. Wheat and its rust parasites in Australia. In Wheat Science-Today and Tomorrow; Cambridge University Press: Cambridge, UK, 1981; pp. 129-147.

3. Park, R.; Karaoglu, H.; Wallwork, H. Exotic Wheat Leaf Rust Pathotype Detected in South Australia; 2014 Cereal Rust Report; Plant Breeding Institute, University of Sydney: Sydney, Australia, 2014; p. 3. Available online: http://sydney.edu.au/agriculture/documents/pbi/cereal_rust_report_2014.pdf (accessed on 15 February 2017).

4. Bariana, H.S.; Brown, G.N.; Bansal, U.K.; Miah, H.; Standen, G.E.; Lu, M. Breeding triple rust resistant wheat cultivars for Australia using conventional and marker assisted selection technologies. Aust. J. Agric. Res. 2007, 58, 576-587. [CrossRef]

5. Bariana, H.S.; Miah, H.; Brown, G.N.; Willey, N.; Lehmensiek, A. Molecular mapping of durable rust resistance in wheat and its implication in breeding. In Wheat Production in Stressed Environments: Proceedings of the 7th International Wheat Conference, 27 November-2 December 2005, Mar del Plata, Argentina; Buck, H.T., Nisi, J.E., Salomón, N., Eds.; Springer: Dordrecht, The Netherlands, 2007; pp. 723-728. 
6. Bariana, H.S.; Bansal, U.K. Breeding for disease resistance. In Encyclopedia of Applied Plant Sciences, 2nd ed.; Murray, B.G., Murphy, D.J., Eds.; Academic Press: Oxford, UK, 2017; pp. 69-76.

7. Bariana, H.S.; McIntosh, R.A. Genetics of adult plant stripe rust resistance in four Australian wheats and the French cultivar 'Hyhride-de-Bersee'. Plant Breed. 1995, 114, 485-491. [CrossRef]

8. Singh, R.; Huerta-Espino, J.; Rajaram, S.; Barna, B.; Kiraly, Z. Achieving near-immunity to leaf and stripe rusts in wheat by combining slow rusting resistance genes. Acta Phytopathol. Entomol. Hung. 2000, 35, 133-139.

9. Jeger, M.J.; Viljanen-Rollinson, S.L.H. The use of the area under the disease-progress curve (AUDPC) to assess quantitative disease resistance in crop cultivars. Theor. Appl. Genet. 2001, 102, 32-40. [CrossRef]

10. Kuhn, R.; Ohm, H.; Shaner, G. Slow leaf-rusting resistance in wheat against twenty-two isolates of Puccinia recondita. Phytopathology 1978, 68, 651-656. [CrossRef]

11. Shaner, G. Growth of uredinia of Puccinia recondita in leaves of slow- and fast-rusting wheat cultivars. Phytopathology 1983, 73, 931-935. [CrossRef]

12. Ayliffe, M.; Periyannan, S.K.; Feechan, A.; Dry, I.; Schumann, U.; Wang, M.-B.; Pryor, A.; Lagudah, E. A simple method for comparing fungal biomass in infected plant tissues. MPMI 2013, 26, 658-667. [CrossRef]

13. Li, Z.; Lan, C.; He, Z.; Singh, R.P.; Rosewarne, G.M.; Chen, X.; Xia, X. Overview and application of QTL for adult plant resistance to leaf rust and powdery mildew in wheat. Crop Sci. 2014, 54, 1907-1925. [CrossRef]

14. Bansal, U.K.; Arief, V.N.; DeLacy, I.H.; Bariana, H.S. Exploring wheat landraces for rust resistance using a single marker scan. Euphytica 2013, 194, 219-233. [CrossRef]

15. Daetwyler, H.D.; Bansal, U.K.; Bariana, H.S.; Hayden, M.J.; Hayes, B.J. Genomic prediction for rust resistance in diverse wheat landraces. Theor. Appl. Genet. 2014, 127, 1795-1803. [CrossRef]

16. Dyck, P.L.; Kerber, E.R.; Lukow, O.M. Chromosome location and linkage of a new gene (Lr33) for reaction to Puccinia recondita. Genome 1987, 29, 463-466. [CrossRef]

17. McIntosh, R.A.; Wellings, C.R.; Park, R.F. Wheat Rusts, an Atlas of Resistance Genes; CSIRO Publications: East Melbourne, Victoria, Australia, 1995.

18. Chhetri, M.; Bansal, U.; Toor, A.; Lagudah, E.; Bariana, H. Genomic regions conferring resistance to rust diseases of wheat in a W195/BTSS mapping population. Euphytica 2016, 209, 637-649. [CrossRef]

19. Bansal, U.K.; Kazi, A.G.; Singh, B.; Hare, R.A.; Bariana, H.S. Mapping of durable stripe rust resistance in a durum wheat cultivar Wollaroi. Mol. Breed. 2014, 33, 51-59. [CrossRef]

20. Wang, S.; Wong, D.; Forrest, K.; Allen, A.; Chao, S.; Huang, B.E.; Maccaferri, M.; Salvi, S.; Milner, S.G.; Cattivelli, L. Characterization of polyploid wheat genomic diversity using a high-density 90,000 single nucleotide polymorphism array. Plant Biotech. J. 2014, 12, 787-796. [CrossRef]

21. Cavanagh, C.R.; Chao, S.; Wang, S.; Huang, B.E.; Stephen, S.; Kiani, S.; Forrest, K.; Saintenac, C.; Brown-Guedira, G.L.; Akhunova, A. Genome-wide comparative diversity uncovers multiple targets of selection for improvement in hexaploid wheat landraces and cultivars. Proc. Natl. Acad. Sci. USA 2013, 110, 8057-8062. [CrossRef]

22. Manly, K.F.; Cudmore, R.H., Jr.; Meer, J.M. Map Manager QTX, cross-platform software for genetic mapping. Mamm. Genome 2001, 12, 930-932. [CrossRef]

23. Kosambi, D. The estimation of map distances from recombination values. Ann. Eugen. 1943, 12, $172-175$. [CrossRef]

24. Wang, S.; Basten, C.J.; Zeng, Z.B. Windows QTL Cartographer 2.5; Department of Statistics, North Carolina State University: Raleigh, NC, USA, 2012.

25. Lagudah, E.S.; McFadden, H.; Singh, R.P.; Huerta-Espino, J.; Bariana, H.S.; Spielmeyer, W. Molecular genetic characterization of the $L r 34 / Y r 18$ slow rusting resistance gene region in wheat. Theor. Appl. Genet. 2006, 11, 21-30. [CrossRef]

26. Herrera-Foessel, S.A.; Singh, R.P.; Huerta-Espino, J.; Rosewarne, G.M.; Periyannan, S.K.; Viccars, L.; Calvo-Salazar, V.; Lan, C.; Lagudah, E.S. Lr68: A new gene conferring slow rusting resistance to leaf rust in wheat. Theor. Appl. Genet. 2012, 124, 1475-1486. [CrossRef]

27. Wright, S. Evolution and genetics of populations. In Genetics and Biometric Foundations; University of Chicago Press: Chicago, IL, USA, 1968; Volume 1.

28. Mago, R.; Miah, H.; Lawrence, G.J.; Wellings, C.R.; Spielmeyer, W.; Bariana, H.S.; McIntosh, R.A.; Pryor, A.J.; Ellis, J.G. High-resolution mapping and mutation analysis separate the rust resistance genes Sr31, Lr26 and Yr9 on the short arm of rye chromosome 1. Theor. Appl. Genet. 2005, 112, 41-50. [CrossRef] 
29. Dyck, P.L.; Sykes, E.E. Genetics of leaf-rust resistance in three spelt wheats. Can. J. Plant Sci. 1994, 74, $231-233$. [CrossRef]

30. Singh, R.; Mujeeb-Kazi, A.; Huerta-Espino, J. Lr46: A gene conferring slow-rusting resistance to leaf rust in wheat. Phytopathology 1998, 88, 890-894. [CrossRef] [PubMed]

31. Helguera, M.; Vanzetti, L.; Soria, M.; Khan, I.A.; Kolmer, J.; Dubcovsky, J. PCR markers for Triticum speltoides leaf rust resistance gene $L r 51$ and their use to develop isogenic hard red spring wheat lines. Crop Sci. 2005, 45, 728-734. [CrossRef]

32. McIntosh, R.A.; Devos, K.M.; Dubcovsky, J.; Rogers, W.J.; Morris, C.F.; Appels, R.; Anderson, O.D. Catalogue of Gene Symbols for Wheat: 2005 Supplement. pp. 1-56. Available online: https://shigen.nig.ac.jp/wheat/ komugi/genes/macgene/supplement2005.pdf (accessed on 25 June 2020).

33. Singh, D.; Mohler, V.; Park, R.F. Discovery, characterisation and mapping of wheat leaf rust resistance gene Lr71. Euphytica 2012, 190, 131-136. [CrossRef]

34. Singla, J.; Lüthi, L.; Wicker, T.; Bansal, U.; Krattinger, S.G.; Keller, B. Characterization of Lr75: A partial, broad-spectrum leaf rust resistance gene in wheat. Theor. Appl. Genet. 2017, 130, 1-12. [CrossRef] [PubMed]

35. Mateos-Hernandez, M.; Singh, R.P.; Hulbert, S.H.; Bowden, R.L.; Huerta-Espino, J.; Gill, B.S.; Brown-Guedira, G. Targeted mapping of ESTs linked to the adult plant resistance gene Lr46 in wheat using synteny with rice. Funct. Integr. Genom. 2005, 6, 122-131. [CrossRef]

36. Kumar, S.; Phogat, B.S.; Vikas, V.K.; Sharma, A.K.; Saharan, M.S.; Singh, A.K.; Kumari, J.; Singh, R.; Jacob, S.R.; Singh, G.P.; et al. Mining of Indian wheat germplasm collection for adult plant resistance to leaf rust. PLOS ONE 2019. [CrossRef]

37. Rosewarne, G.M.; Singh, R.P.; Huerta-Espino, J.; Herrera-Foessel, S.A.; Forrest, K.L.; Hayden, M.J.; Rebetzke, G.J. Analysis of leaf and stripe rust severities reveals pathotype changes and multiple minor QTLs associated with resistance in an Avocet $\times$ Pastor wheat population. Theor. Appl. Genet. 2012, 124, 1283-1294. [CrossRef]

38. Rosewarne, G.M.; Singh, R.P.; Huerta-Espino, J.; Rebetzke, G.J. Quantitative trait loci for slow-rusting resistance in wheat to leaf rust and stripe rust identified with multi-environment analysis. Theor. Appl. Genet. 2008, 116, 1027-1034. [CrossRef]

39. Nsabiyera, V.; Qureshi, N.; Bariana, H.S.; Wong, D.; Forrest, K.L.; Hayden, M.J.; Bansal, U.K. Molecular markers for adult plant leaf rust resistance gene Lr48 in wheat. Mol. Breed. 2016, 36, 1-9. [CrossRef]

40. Buerstmayr, M.; Matiasch, L.; Mascher, F.; Vida, G.; Ittu, M.; Robert, O.; Holdgate, S.; Flath, K.; Neumayer, A.; Buerstmayr, H. Mapping of quantitative adult plant field resistance to leaf rust and stripe rust in two European winter wheat populations reveals co-location of three QTL conferring resistance to both rust pathogens. Theor. Appl. Genet. 2014, 127, 2011-2028. [CrossRef] [PubMed]

41. Faris, J.; Li, W.; Liu, D.; Chen, P.; Gill, B. Candidate gene analysis of quantitative disease resistance in wheat. Theor. Appl. Genet. 1999, 98, 219-225. [CrossRef]

42. Messmer, M.M.; Seyfarth, R.; Keller, M.; Schachermayr, G.; Winzeler, M.; Zanetti, S.; Feuillet, C.; Keller, B. Genetic analysis of durable leaf rust resistance in winter wheat. Theor. Appl. Genet. 2000, 100, 419-431. [CrossRef]

43. Xu, X.; Bai, G.; Carver, B.F.; Shaner, G.E.; Hunger, R.M. Molecular characterization of slow leaf-rusting resistance in wheat. Crop Sci. 2005, 45, 758-765. [CrossRef]

44. Xu, X.Y.; Bai, G.H.; Carver, B.F.; Shaner, G.E.; Hunger, R.M. Mapping of QTLs prolonging the latent period of Puccinia triticina infection in wheat. Theor. Appl. Genet. 2005, 110, 244-251. [CrossRef]

45. Schnurbusch, T.; Paillard, S.; Schori, A.; Messmer, M.; Schachermayr, G.; Winzeler, M.; Keller, B. Dissection of quantitative and durable leaf rust resistance in Swiss winter wheat reveals a major resistance QTL in the Lr34 chromosomal region. Theor. Appl. Genet. 2003, 108, 477-484. [CrossRef]

46. Somers, D.J.; Isaac, P.; Edwards, K. A high-density microsatellite consensus map for bread wheat (Triticum aestivum L.). Theor. Appl. Genet. 2004, 109, 1105-1114. [CrossRef]

(C) 2020 by the authors. Licensee MDPI, Basel, Switzerland. This article is an open access article distributed under the terms and conditions of the Creative Commons Attribution (CC BY) license (http://creativecommons.org/licenses/by/4.0/). 